\title{
Superoxide dismutase gene polymorphism is associated with ischemic stroke risk in the Chinese Han population
}

\author{
Yang Xitong \\ Dali University \\ Yang Sulian \\ Dali University \\ Xu Hongyang \\ Hospital of traditional chinese medicine guangde \\ Zhang Yuanyuan \\ Dali University \\ wang guangming ( $\nabla$ wgm1991@dali.edu.cn ) \\ https://orcid.org/0000-0002-0220-1493
}

Research article

Keywords: Ischemic stroke, Superoxide dismutases, Gene polymorphism, Chinese Han, SOD3 gene, rs7655372 locus polymorphism

Posted Date: October 21st, 2019

DOl: https://doi.org/10.21203/rs.2.16256/v1

License: (c) (i) This work is licensed under a Creative Commons Attribution 4.0 International License. Read Full License 


\section{Abstract}

Background:Stroke is a serious cardiovascular disease and a major cause of disability and death in both developed and developing countries. Superoxide dismutases (SODs) are enzymes that catalyze the breakdown of superoxide into oxygen and hydrogen peroxide and play a key role in the antioxidant response. This study explored the relationship between single nucleotide polymorphisms (SNPs) in SOD genes and the risk of ischemic stroke (IS) in the Chinese Han population of Dali city.

Methods: For this case-control study, we enrolled 144 IS patients and 128 healthy controls. The SNPs rs 17880487 and rs 80265967 of the SOD 1 gene, rs4880 and rs284296 of the SOD2 gene, rs2695232 and rs7655372 of the SOD3 gene were detected via Taqman-polymerase chain reaction (PCR). Genotypes and allele frequencies of the two groups were compared. Odds ratio (OR) and 95\% confidence intervals (Cls) were calculated by unconditional logistic regression, and environmental factors were corrected with multivariate logistic regression analysis.

Results: Rs7655372 of SOD3 was associated with a significantly increased risk of IS. Moreover, this the G and GA genotypes of SNP rs7655372 were associated with an increased risk of IS, whereas the A and GA genotypes were risk factors for IS. Furthermore, multivariate logistic regression analysis showed that fasting blood glucose level, red blood cell count, white blood cell count, low-density lipoprotein level, systolic pressure, diastolic pressure, and rs 7655372 GA/GG were independent risk factors for IS.

Conclusions: The SOD3 gene rs7655372 locus polymorphism is a risk factor for IS, and its frequency in the Chinese Han population of Dali City denotes that this population is at increased risk of IS.

\section{Background}

Ischemic stroke (IS) is one of the most complex cardiovascular diseases associated with high morbidity, disability, death, and recurrence rate[1,2]. According to the World Health Organization, 15 million people suffer strokes worldwide each year, more than 6 million die, and another 5 million are permanently disabled[3,4]. In China, IS is the second leading cause of death and one of the main causes of adult disability[5,6]. IS-associated pain and comorbidities significantly impact the quality of life of patients, as well as increasing the burden on their families and society. Therefore, effective prevention and treatment strategies are urgently needed.

IS is a nervous system disorder with multiple complex factors, including modifiable risk factors (environment) and nonmodifiable risk factors (heredity). The traditional risk factors for IS include smoking, lack of exercise, an unhealthy diet as well as some diseases, such as 
obesity, diabetes, arteriosclerosis, hypertension, atrial fibrillation, and dyslipidemia[7]. Epidemiological studies support that there are genetic factors associated with stroke, and gene polymorphisms may regulate the pathophysiological process of IS[8].

Superoxide dismutases (SODs) are a class of antioxidant enzymes that play a pivotal role in reducing oxidative stress and maintain intracellular and extracellular oxidant/antioxidant balance. SODs exert their effects by catalyzing the dismutation of superoxide into oxygen and hydrogen peroxide and scavenging the oxygen free radicals[9]. Oxidative stress is the excessive accumulation of reactive oxygen species (ROS) and is a major cause of cardiovascular disease[10,11].There are three isoforms of SODs: including the copper-andzinc-containing SODs (CuZn-SOD/ SOD1), which is primarily located in the cytoplasm; manganese SOD (Mn-SOD/ SOD2), which is located in the mitochondria; and extracellular SOD (EC-SOD/SOD3) $[12,13]$.

In a normal physiological state, a major source of ROS would be the mitochondria. ROS is the product of oxidative phosphorylation in the mitochondrial respiratory chain, where it scavenges catalase, glutathione peroxidase, glutathione reductase, and superoxide dismutase via antioxidants[14]. SODs are endogenous enzymes that convert superoxide anions into hydrogen peroxide and oxygen, SOD1 is considered an important cellular enzyme as it plays a key role in the protection of cells from damage caused by superoxide free radicals under stress conditions such as high temperature and humidity. Moreover, SOD1 acts as an antioxidant enzyme which maintains the oxidation/antioxidant balance either intracellularly and extracellularly and catalyzed the mutation of superoxide radicals into oxygen and hydrogen peroxide[15]. SOD2 scavenged about $80 \%$ of the free radicals in the oxidation and phosphorylation processes associated with the mitochondria. Incomplete scavenging of reactive oxygen species leads to increased free radical levels, which could cause lipid peroxidation and severe cell and tissue damage[16]. SOD3, which is encoded by a gene distinct from that of CuZn-SOD, is composed of 240 amino acids and harbors an 18amino-acid-long signal peptide which targeted proteins for the extracellular compartment[17]. 
Recent studies have found that the SODs gene variations are associated with the risk of different diseases, including cardiovascular diseases. The Alachkar study reported that the CC genotype of rs4880 is associated with increasing hepatotoxicity following asparaginasebased treatment[18]. Ghattas reported that in the Egyptian population, individuals with rs2234694 CC genotype showed an increased risk of T2DM[19]. Otaki et al. found, in a study on 2,799 healthy subjects, that rs1041740 and rsl7880487 in the SODl gene were related to cardiovascular mortality[20]. However, the relationship between the SOD gene variations and the risk of IS remains unclear.

Here, we conducted a case-control study to investigate the polymorphisms rs17880487, rs80265967, rs4880, rs284296, rs2695232 and rs7655372 in the SOD genes of 144 IS patients and 128 healthy controls to determine whether these SNPs are associated with increased risk of IS in the Chinese Han population. Our results are expected to contribute valuable insights into the potential role of SOD gene polymorphisms in IS and might help in the development of prevention and targeted treatment strategies for IS.

\section{Methods}

Study Subjects

The study subjects consisted of 144 IS patients (80 males, 64 females) and 128 control patients (68 males, 60 females), who were recruited from outpatient and inpatient services in the first affiliated hospital of Dali University from August 2017 to August 2018. The diagnosis of IS was based on the WHO standards of thrombotic patients with cerebral infarction and neurological abnormalities confirming thrombotic cerebral infarction with CT or MRI. The inclusion criteria for the IS patient group were as follows: (1) IS diagnosis was according to the diagnostic criteria of cerebrovascular diseases from the Fourth National Conference; (2) The individual and their family were residents of Dali City for more than three generations. Patients with the following conditions were excluded hemorrhagic stroke, transient ischemic attack, cerebrovascular malformation, and IS caused by trauma. The control group individuals were free of cardiovascular and cerebrovascular diseases, autoimmune disorders, malignant tumors, immunological 
diseases, neurological deficits, severe hepatic, and renal dysfunction. Furthermore, there was no sibship between the selected control subjects and the IS patients or study subjects from the Dali region who had lived there for over three generations. In this study, some clinical data were collected, such as age, sex, fasting blood glucose (FBG), red blood cells (RBC), white blood cells (WBC), cholesterol (TCH), triglycerides (TG), low density lipoprotein (LDL), systolic pressure (SPB), diastolic pressure (DBP), and body mass index (BMI).The study protocol was approved by the Medical Ethics Committee of the first affiliated hospital of Dali University, and all participants provided informed consent.

SNP selection and genotyping

About $5 \mathrm{ml}$ of peripheral blood samples was collected into tubes containing ethylenediamine tetraacetic acid (EDTA). Genomic DNA was extracted according to the manufacturer's protocols (Bomaide Technology, Beijing, China) and stored at $-80^{\circ} \mathrm{C}$ until analysis. Six SNPs of SODs were genotyped with Taqman polymerase chain reaction (Taqman-PCR).

According to the NCBI database, six SNPs rs1780487, rs80265967, rs4880, rs2842960, rs2695232, and rs7655372 of SODs were selected. The amplification was performed in a 25 $\mu \mathrm{L}$ volume, $12.5 \mu \mathrm{l} 2 \times \mathrm{Taq}$ enzyme mixture, $0.5 \mu \mathrm{l}$ CF forward primer, $0.5 \mu \mathrm{l}$ TF forward

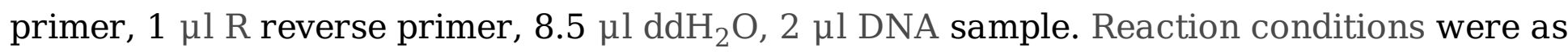
follows: predegeneration at $95^{\circ} \mathrm{C}$ for $5 \mathrm{~min}$, denaturation at $95^{\circ} \mathrm{C}$ for $10 \mathrm{~s}$, annealing at 60 ${ }^{\circ} \mathrm{C}$ for $30 \mathrm{~s}$, followed by 30 cycles of extension at $72^{\circ} \mathrm{C}$ for $2 \mathrm{~min}$ and conservation at $16{ }^{\circ} \mathrm{C}$ for 5 min. Primers were designed using the Primer 5 software. The sequences of the primers and probes from Anhui General Biosystems are listed in Table 1.

Table 1 Primer sequences used in this study 


\begin{tabular}{|c|c|c|}
\hline SNP & primer & Sequence \\
\hline \multirow[t]{3}{*}{ rs17880487 } & $\mathrm{CC}$ & 5'-TATTATGAGGCTATTAAAAGAATCC-3' \\
\hline & TT & 5'-TATTATGAGGCTATTAAAAGAATCTT-3' \\
\hline & Reverse & 5'-ATCAGAGCTAATTTAGTTTGAATTT-3' \\
\hline \multirow[t]{3}{*}{ rs80265967 } & $\mathrm{AA}$ & 5'-GAGACTTGGGCAATGTGACTGCTGA-3' \\
\hline & $\mathrm{CC}$ & 5'-GAGACTTGGGCAATGTGACTGCTGC-3' \\
\hline & Reverse & 5'-AGACACATCGGCCACACCATCTTTG-3' \\
\hline \multirow[t]{3}{*}{ rs 4880} & $\mathrm{CC}$ & 5'-TGCCTGGAGCCCAGATACCCCAAAG-3' \\
\hline & TT & 5'-TGCCTGGAGCCCAGATACCCCAAAA-3' \\
\hline & Reverse & 5'-AGCACCAGCAGGCAGCTGGCTCCGG-3' \\
\hline \multirow[t]{3}{*}{ rs2842960 } & $\mathrm{CC}$ & 5'-GAGACCAAACATTTCCCCAAAGCAC-3' \\
\hline & $\mathrm{TT}$ & 5'-GAGACCAAACATTTCCCCAAAGCAT-3' \\
\hline & Reverse & 5'-ATTGAAATTGTTCTTGTTGTATAAT-3' \\
\hline \multirow[t]{3}{*}{ Rs2695232 } & $\mathrm{CC}$ & 5'-ТTCTCСТСТGCTCCAACAGACACCC-3' \\
\hline & $\mathrm{TT}$ & 5'-ТTCTCСТCTGCTCCAACAGACACCT-3' \\
\hline & Reverse & 5'-GGCGAAGGTGAGACCTCAGAGTGGA-3' \\
\hline \multirow[t]{3}{*}{ Rs7655372 } & AA & 5'-TGCCCTAGATGAGAGATGTGCAGTA-3' \\
\hline & GG & 5'-TGCCCTAGATGAGAGATGTGCAGTG-3' \\
\hline & Reverse & 5'-CTCCAAGCCCAAGGTTTAGGCACCA-3' \\
\hline
\end{tabular}

\section{Statistical analyses}

The statistical analyses were performed using SPSS 19.0 software. Hardy-Weinberg equilibrium (HWE) was assessed by HWE software for SOD SNP genotype distribution in the controls. A p-value匹0.05 indicated a balanced genetic and Mendelian population. Count data and quantitative data were processed using t-test and $\chi^{2}$-test, respectively. Multivariate logistic regression analysis were performed after adjusting for Age, gender, FBG, WBC, RBC, TC, TG, LDL, SBP, DBP, and BMI to test the correlation between gene variation and risk of IS. Odds ratio (OR) and 95\% confidence interval (CI) were calculated 
using unconditional logistic regression, and co-dominant, dominant, recessive, and additive were used to assess these relationships. A p-value $<0.05$ was considered significant.

\section{Results}

Clinical characteristics

The main clinical characteristics of the control and IS groups are summarized in Table 2 .

Significant differences between the groups were found in the following tests: FBG,WBC, RBC, TC, TG, LDL, and SBP. However, no significant differences were noted in the age, sex, DBP, or BMI ( Table 2).

Table2 characteristics of ischemic stroke patients and controls

\begin{tabular}{|c|c|c|c|}
\hline Characteristics & controls & IS patients & $\mathrm{P}$ \\
\hline $\operatorname{age}(y, m e a n \pm S D)$ & $63.82 \pm 8.69$ & $67.18 \pm 4.71$ & $\square 0.05$ \\
\hline Male gender, ( $\mathrm{n})$ & 68 & 90 & $\square 0.05$ \\
\hline $\mathrm{FBG}(\mathrm{mmol} / \mathrm{L}, \mathrm{mean} \pm \mathrm{SD})$ & $6.25 \pm 2.73$ & $4.75 \pm 0.87$ & $\square 0.05^{\mathrm{a}}$ \\
\hline $\operatorname{WBC}\left(10^{\wedge} 12 / \mathrm{L}\right.$, , mean $\left.\pm \mathrm{SD}\right)$ & $6.27 \pm 1.57$ & $7.79 \pm 2.32$ & $00.05^{\mathrm{a}}$ \\
\hline $\mathrm{RBC}\left(10^{\wedge} 12 / \mathrm{L}, \mathrm{mean} \pm \mathrm{SD}\right)$ & $4.97 \pm 0.55$ & $4.81 \pm 0.72$ & $00.05^{\mathrm{a}}$ \\
\hline $\mathrm{TC}(\mathrm{mmol} / \mathrm{L} \square \mathrm{mean} \pm \mathrm{SD})$ & $4.33 \pm 0.42$ & $5.01 \pm 1.29$ & $00.05^{\mathrm{a}}$ \\
\hline $\mathrm{TG}(\mathrm{mmol} / \mathrm{L} \square \mathrm{mean} \pm \mathrm{SD})$ & $1.35 \pm 0.75$ & $1.72 \pm 1.40$ & $\square 0.05^{\mathrm{a}}$ \\
\hline $\mathrm{LDL}(\mathrm{mmol} / \mathrm{L} \square$ mean $\pm \mathrm{SD})$ & $2.82 \pm 0.72$ & $2.82 \pm 0.72$ & $00.05^{\mathrm{a}}$ \\
\hline $\mathrm{SBP}(\mathrm{mmHg}$, mean $\pm \mathrm{SD})$ & $144.92 \pm 25.88$ & $133.85 \pm 15.34$ & $\square 0.05^{\mathrm{a}}$ \\
\hline $\mathrm{DBP}(\mathrm{mmHg}, \mathrm{mean} \pm \mathrm{SD})$ & $86.46 \pm 13.02$ & $86.46 \pm 13.02$ & $\square 0.05$ \\
\hline $\operatorname{BMI}\left(\mathrm{kg} / \mathrm{m}^{2}\right.$, mean $\left.\pm \mathrm{SD}\right)$ & $84.656 \pm 10.40$ & $83.15 \pm 3.28$ & 00.05 \\
\hline
\end{tabular}

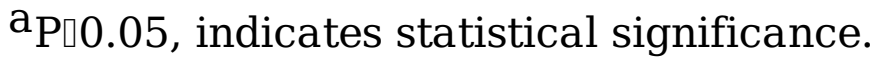


Hardy-Weinberg balance analysis

The genotype frequencies of six SNPs followed Hardy-Weinberg equilibrium in the control subjects ( $\mathrm{P}>0.05$ for six SNPs), these results indicated a balanced genetic and Mendelian population, the results as demonstrated in Table 3.

Table 3 Hardy-Weinberg results of control and IS

\begin{tabular}{llllll}
\hline SNP & \multicolumn{2}{c}{$\chi^{2}$} & & \multicolumn{2}{c}{$\mathrm{P}$} \\
\cline { 2 - 3 } \cline { 5 - 6 } & Control & IS & & Control & IS \\
\hline rs17880487 & 0.258 & 0.347 & & 0.611 & 0.556 \\
rs4880 & 0.21 & 0.55 & 0.65 & 0.46 \\
rs2842960 & 0.68 & 0.23 & 0.41 & 0.63 \\
rs2695232 & 4.078 & 0.155 & 0.053 & 0.693 \\
rs7655372 & 0.07 & 0.589 & 0.786 & 0.442 \\
\hline
\end{tabular}

SODs polymorphism and risk of IS

The genotype distributions of six SNPs of SOD genes between the IS and controls and their association with IS are summarized in Table 4.

Rs80265967 of SOD1 gene showed no polymorphism, while the other five sites showed polymorphism. Different genetic models were used to analyze the association between the tested SNPs and the risk of IS in the five loci of the SOD genes (Table 4). Allele A and genotype GA of rs7655372 were related to significantly increased risk of IS. In the Logadditive, GA/GG was associated with a 2.722 -fold increase in IS risk (OR=2.722, 95\%CI= 1.039-7.132, $\mathrm{p}=0.042$, adjusted OR 5.128, 95\% CI 1.558-16.881, $\mathrm{p}=0.007$ ). The allele A/G was associated with a 2.722-fold increase in IS risk $(\mathrm{OR}=2.722,95 \% \mathrm{CI}=1.039-7.132$, $\mathrm{p}=0.042$, adjusted $\mathrm{OR}=5.128,95 \% \mathrm{CI}=1.558-16.881, \mathrm{p}=0.007)$. Following adjustments for 
age, sex, FBG, RBC, WBC, TC, TG, LDL, and SBP, the allele GA/GG was found to increase the risk of stroke $(\mathrm{P}=0.007)$.

Independent risk factors were identified for IS in rs7655372 by multivariate logistic regression analysis. As depicted in Table 5, the independent risk factors were FBG (OR= $3.006,95 \%$ CI 1.988-4.543), RBC (OR=0.607, 95\% CI 0.375-0.984), WBC $(\mathrm{OR}=1.488 ; 95 \%$ CI $0.615-2.308)$, LDL $(\mathrm{OR}=1.191 ; 95 \%$ CI $0.615-2.308)$, TC $(\mathrm{OR}=1.191 ; 95 \%$ CI $0.615-$ 2.308), SB P (OR $=1.191 ; 95 \%$ CI 0.615-2.308), DBP (OR $=1.191 ; 95 \%$ CI 0.615-2.308), and GA/GG genotype $(\mathrm{OR}=5.128 ; 95 \%$ CI 1.558-16.881) .

Table 4 Genotype frequencies of SODs gene polymorphisms in cases and controls and their associations with IS 


\begin{tabular}{|c|c|c|c|c|c|c|c|}
\hline$\lceil\mathrm{P}$ & genotype & Case[n(\%)] & Control[n(\%)] & $\mathrm{OR}(95 \mathrm{CI})$ & $\mathrm{P}$ & Adjusted OR(95CI) & $\begin{array}{l}\text { Adjusted } \\
\text { P }\end{array}$ \\
\hline \multirow[t]{5}{*}{0487} & $\mathrm{CC}$ & $131(90.97 \%)$ & $117(91.41 \%)$ & reference & reference & & \\
\hline & $\mathrm{CT}$ & $13(9.03 \%)$ & $11(8.59 \%)$ & $1.056(0.455,2.447)$ & 0.900 & $0.879(0.277,2.789)$ & 0.827 \\
\hline & $\mathrm{TT}$ & 0 & 0 & & & & \\
\hline & $\mathrm{C}$ & $275(95.49 \%)$ & $245(95.70 \%)$ & reference & reference & & \\
\hline & $\mathrm{T}$ & $13(4.51 \%)$ & $11(4.30 \%)$ & $1.056(0.455,2.447)$ & 0.900 & $0.879(0.277,2.789)$ & 0.827 \\
\hline$\$ 5967$ & AA & $155(100 \%)$ & $122(100 \%)$ & -- & -- & -- & -- \\
\hline \multirow[t]{3}{*}{380} & $\mathrm{TT}$ & $105(72.92 \%)$ & $96(75 \%)$ & reference & reference & & \\
\hline & $\mathrm{TC}$ & $37(25.69 \%)$ & $29(22.66 \%)$ & $1.167(0.667,2.041)$ & 0.589 & $1.045(0.486,2.250)$ & 0.91 \\
\hline & $\mathrm{CC}$ & $2(1.39 \%)$ & $3(2.34 \%)$ & $0.610 \square 0.10,3.726 \square$ & 0.592 & $0.86(0.104,7.108)$ & 0.889 \\
\hline \multirow[t]{2}{*}{ nant } & $\mathrm{CC}+\mathrm{CT}$ & & & $1.114 \square 0.647,1.918 \square$ & 0.696 & $1.026(0.490,2.149)$ & 0.945 \\
\hline & vs TT & & & & & & \\
\hline \multirow[t]{4}{*}{ ssive } & CC vs & & & $0.587(0.096,3.569)$ & 0.563 & $0.851(0.104,6.974)$ & 0.88 \\
\hline & $\mathrm{CT}+\mathrm{TT}$ & & & & & & \\
\hline & $\mathrm{T}$ & $247(85.76 \%)$ & $221(86.33 \%)$ & reference & reference & & \\
\hline & $\mathrm{C}$ & $41(14.24 \%)$ & $35(13.67 \%)$ & $1.049(0.643,1.709)$ & 0.849 & $1.005(0.527,1.914)$ & 0.989 \\
\hline \multirow[t]{3}{*}{2960} & $\mathrm{CC}$ & $106(73.61 \%)$ & $94(73.43 \%)$ & reference & reference & & \\
\hline & $\mathrm{CT}$ & $34(23.61 \%)$ & $30(23.44 \%)$ & $1.005(0.572,1.767)$ & 0.986 & $0.947(0.438,2.047)$ & 0.889 \\
\hline & $\mathrm{TT}$ & $4(2.78 \%)$ & $4(3.13 \%)$ & $0.887(0.261,3.645)$ & 0.868 & $0.868(0.128,5.886)$ & 0.885 \\
\hline \multirow[t]{2}{*}{ nant } & $\mathrm{TT}+\mathrm{TC}$ & & & $0.991(0.578,1.700)$ & 0.974 & $0.963(0.462,2.007)$ & 0.921 \\
\hline & vs CC & & & & & & \\
\hline \multirow[t]{4}{*}{ ssive } & TT vs & & & $0.886(0.217,3.616)$ & 0.866 & $0.105(0.179,6.832)$ & 0.915 \\
\hline & $\mathrm{TC}+\mathrm{CC}$ & & & & & & \\
\hline & $\mathrm{C}$ & $246(85.42 \%)$ & $226(85.61 \%)$ & reference & reference & & \\
\hline & $\mathrm{T}$ & $42(14.58 \%)$ & $38(14.39 \%)$ & $0.981(0.618,1.555)$ & 0.934 & $0.985(0.531,1.829)$ & 0.962 \\
\hline \multirow[t]{3}{*}{5232} & $\mathrm{CC}$ & $53(36.81 \%)$ & $44(34.37 \%)$ & reference & reference & & \\
\hline & $\mathrm{CT}$ & $67(46.53 \%)$ & $71(55.47 \%)$ & $0.783(0.465,1.319)$ & 0.358 & $1.024(0.513,1.829)$ & 0.962 \\
\hline & $\mathrm{TT}$ & $24(16.66 \%)$ & 13(10.16\%) & $1.533(0.699,3.358)$ & 0.286 & $1.334(0.475,3.742)$ & 0.585 \\
\hline
\end{tabular}




\begin{tabular}{|c|c|c|c|c|c|c|c|}
\hline ant & $\mathrm{TT}+\mathrm{TC}$ & & & $0.899(0.547,1.479)$ & 0.676 & $1.083(0.560,2.094)$ & 0.813 \\
\hline & vs CC & & & & & & \\
\hline ive & TT vs & & & $1.769(0.860,3.641)$ & 0.121 & $1.316(0.506,3.423)$ & 0.574 \\
\hline & $\mathrm{TC}+\mathrm{CC}$ & & & & & & \\
\hline & $\mathrm{C}$ & $173(60.07 \%)$ & $159(62.11 \%)$ & reference & reference & & 0.65 \\
\hline & $\mathrm{T}$ & $115(39.93 \%)$ & 97(37.89\%) & $1.096(0.767,1.567)$ & 0.614 & $1.117(0.692,1.803)$ & 0.65 \\
\hline 5372 & GG & $127(88.19 \%)$ & $122(95.31 \%)$ & reference & reference & & \\
\hline & GA & $17(11.81 \%)$ & $6(4.69 \%)$ & $2.722(1.039,7.132)$ & $0.042^{*}$ & $5.128(1.558,16.881)$ & $0.007^{*}$ \\
\hline & $\mathrm{AA}$ & 0 & 0 & & & & \\
\hline & G & $271(94.10 \%)$ & $250(97.66 \%)$ & reference & reference & & \\
\hline & $\mathrm{A}$ & $17(5.90 \%)$ & $6(2.34 \%)$ & $2.722(1.039,7.132)$ & $0.042^{*}$ & $5.128(1.558,16.881)$ & $0.007^{*}$ \\
\hline
\end{tabular}

ORs: odds ratios; CI: confidence interval; ${ }^{*}$ p value 0.05 indicates statistical significance. Adjusted by age, sex, fasting blood glucose, red blood cells, white blood cells, cholesterol (TCH), triglycerides(TG), low density lipoprotein (LDL), systolic pressure (SPB ) and diastolic pressure (DBP).

Table 5 Rs7655372 multivariate logistic regression analysis of independent risk factors for IS 


\begin{tabular}{ccccc}
\hline Variables & OR & 95\%CI & $\mathrm{P}$ \\
\cline { 1 - 2 } sex & 1.191 & $0.615-2.308$ & 0.604 \\
age & 1.013 & $0.986-1.041$ & 0.34 \\
FBG & 3.006 & $1.988-4.543$ & 00.001 \\
RBC & 0.607 & $0.375-0.984$ & 0.043 \\
WBC & 1.488 & $1.245-1.779$ & $\square 0.001$ \\
LDL & 1.543 & $1.06-2.246$ & 0.023 \\
TC & 1.754 & $1.19-2.586$ & 0.005 \\
TG & 1.554 & $0.981-2.462$ & 0.061 \\
SBP & 1.022 & $1.006-1.039$ & 0.007 \\
DBP & 0.976 & $0.954-1.000$ & 0.048 \\
GA/GG & 5.128 & $1.558-16.881$ & 0.007 \\
\hline
\end{tabular}

$\mathrm{CI}=$ confidence interval

\section{Discussion}

IS is a complex neurological disease caused by many factors, which is related to environmental factors and genetic factors, and gene variation has become one of the important factors of ischemic stroke. This cerebrovascular disease is related to age, sex, and genetic factors, and is directly related to hypertension, smoking, diabetes, hyperlipidemia, coronary heart disease, hyperhomocysteine, and other factors[21]. Recent epidemiological studies suggested that IS was closely related to genetic factors. Single nucleotide polymorphisms (SNPs) are the most common types of deoxyribonucleic acid (DNA) variants in humans, caused by the stable substitution of a single nucleotide from a point mutation in the genome. Today, the latest advances in molecular genetics have allowed people to realize that a single nucleotide polymorphism was closely related to the pathogenesis of a stroke[22]. Ischemic stroke results in increased levels of reactive oxygen species (ROS) such as superoxide anions $\left(\mathrm{O}^{2-}\right)$, hydroxyl radical $\left(\mathrm{OH}^{-}\right)$, and hydrogen peroxide $\left(\mathrm{H}_{2} \mathrm{O}_{2}\right)$ in the blood. Increased ROS levels are associated with reperfusion 
injury[23]. ROS damaged cellular proteins, lipids, and DNA, and disrupted normal cellular signaling and gene regulation[24].

In the case-control study, six SNPs of SOD genes (rs17880487, rs80265967, rs4880, rs284296, rs2695232, and rs7655372) were investigated to determine their association with the risk of IS in the Chinese Han population. SNP rs7655372 was found to be associated with an increased risk of IS; however, no significant relationship was found between IS and the other investigated SNPs. Six sites were included: rs17880487 and rs80265967 in the SOD1 gene, rs4880 and rs2842960 in the SOD2 gene, and rs2695232 and rs7655372 in the SOD3 gene.

Rs17880487 is located on chromosome 21:31668917, presenting a 3 prime UTR variant and a downstream transcript variant. Rs80265967 is located on chromosome 21:31667290, presenting a missense variant and a coding sequence variant. Otaki ${ }^{16}$ reported that rsl7880487 was associated with cardiovascular mortality, but the present study showed no correlation between the rsl7880487 and IS risk in the Chinese Han population of Dali City. Furthermore, rs80265967 did not exhibit polymorphisms in this study.

Rs4880 is located on chromosome 6:159692840, presenting a coding sequence variant, missense variant, and 5 prime UTR variant. rs2842960 is located on chromosome 6:159692289, presenting an intron variant and a 3 prime UTR variant. rs4880 and rs2842960 loci are located in the promoter region of the SOD2 gene. Promoter region SNPs could alter gene expression, leading to the development of various diseases. The spatial conformation of a signal peptide could be changed by the rs4880 mutation of the SOD2 gene, thus reducing the rate of transport to the mitochondria and the entry of SOD2 into the mitochondria, where it plays an antioxidant role. This disruption of SOD2 transport increases oxidative stress, leading to cardiovascular disease. The mutation of this site is $\mathrm{C} \rightarrow \mathrm{T}$ could change alanine (Ala) to valine (Val) in the position of the signal peptide, at this point, the signal peptide space transformed from $\alpha$ helix to $\beta$ fold, $\alpha$ helix is amphiphilic, which induced SOD2 from the cytoplasm to the mitochondria, and $\beta$ folding affected the proper identification of the signal peptides and related receptors on the mitochondrial inner 
membrane, and exhibited reduced the transcriptional activity of SOD2 to the mitochondria by $30-40 \%$ affect the entry of SOD2 into mitochondria to play an antioxidant role, which increased the risk of coronary artery disease[25].Mutations in the SOD2 gene increase the risk of developing cancer, SOD2 SNP rs4880 ( $\mathrm{T}>\mathrm{C}$ ) resulted in conformational changes of the protein helix structure, an increased risk of oral cancer, and has been linked to a variety of others cancers[26]. Studies have shown that rs4880 SNP ( $\mathrm{T}>\mathrm{C}$ changes at the nucleotide level) at codon 16 causes alanine (GCT) to replace valine (GTT)[27]. The C allele of SOD2 rs4880 has been reported to retain the protein helix structure of many diseases, and maintained the normal activity of the enzymes, related to Alzheimer's disease[28], however, the polymorphism of rs2842960 (C > T) has been rarely studied, and therefore the association with disease is unclear. In the present study, our results showed that there was not a significant association between rs4880, rs2842960 polymorphism, and IS in the Chinese Han population of Dali City.

Rs2695232 is located on chromosome 4:24800327, presenting a non-coding transcript variant and a 3 prime UTR variant. rs7655372 is located on chromosome 4:24797264, presenting an intron variant. Currently, there are no reports of these two SNPs being associated with diseases. However, studies have shown that the polymorphism of the other SOD3 locus was correlated with disease. The SOD3 Ala40Thr missense mutation (GCGACG) was associated with susceptibility to type 2 diabetes by increasing the risk of type 2 diabetes[29]. Takahiro studied the correlation between the polymorphism of SOD3 and cerebral infarction. The frequency of C-C-C haplotype in the female SOD3 polymorphisms (rs13306703, rs699473, and rs1799895) was significantly higher in patients with cerebral infarction than in the control group. Therefore, SOD3 haploid C-C-C might be a marker of female cerebral infarction[30]. In this study, the rs7655372 A allele and GA genotype increased the risk of IS, whereas A and GA were risk factors for IS. Polymorphisms of the other loci had no correlation with the risk of IS. We analyzed the genotype of rs7655372 (Gם A) locus and found that there was no homozygous AA; therefore, allele (A/G) and additive (GA/GG) were selected for analysis. The allele testing and the additive model indicated rs7655372 increased the risk of IS 2.722-fold. After adjusting the risk factors, A and GA of 
rs7655372 were still found to be risk factors for IS. In addition to traditional stroke risk factors such as hypertension, hyperlipidemia, and diabetes, this SNP might be an independent risk factors for stroke. However, no data about the relationship between rs7655372 and the risk of IS has been reported.

Our results indicate that polymorphisms of rs7655372 increase the risk of IS in the Chinese Han population. However, this study has some limitations. First, our samples were generated from the Han Chinese population of Dali City, and hence the findings are cannot be applicable to other ethnicities. In addition, regional disparity could lead to possible inconsistencies in the role of the SNPs of the same locus in similar diseases of different ethnic groups and different diseases of the same ethnic group.

\section{Conclusions}

We found that the association of rs7655372 of SOD3 with the risk of IS in the Chinese Han population and that the rs7655372 allele A and GA genotype significantly increased the risk of IS. The findings provide valuable insights for future explorations of IS pathogenesis, which could enable the development of prevention, early detection, and treatment strategies.

\section{Declarations}

\section{Additional files}

NCBI database (https://www.ncbi.nlm.nih.gov/snp/).

\section{Abbreviations}

IS: Ischemic stroke;SODS:Superoxide dismutases, SNP:single nucleotide polymorphism CIs: 95\% confidence intervals; OR: Odds ratio; 
The study protocol was approved by the Ethic Committee of Dali University approved the use of human blood samples for this study.All the patients were informed to the subjects and written informed consent was obtained.

\section{Consent for publication}

Not applicable.

\section{Availability of data and materials}

The datasets used or analyzed during the current study are available from the corresponding author on reasonable request.

The datasets supporting the conclusions of this article are included within the article.

\section{Competing interests}

The authors declare that they have no competing interests.

\section{Funding}

This study was supported by National Natural Science Foundation of China(No. 81360206); Scientific research fund project of Yunnan education department (2019J0775);

\section{Authors' contributions}

YXT designed the experiments, draft the manuscript.

YSL and XHY analyzed the data.

YXT and XHY collect specimen.

ZYY and WGM revising the manuscript.

All authors agreed to submit the manuscript for publication. 


\section{Acknowledgements}

We thank all patients in this study and all the research staff for their contributions to this project.

\section{Authors' information (optional)}

1.The First Affiliated hospital of Dali University,Dali 671000, Yunnan, ,China.

2. Hospital of Traditional Chinese Medicine,Guangde,242200,Anhui, China.

\section{References}

1. Zhang $Y$, Wang $X, X u X$, et al.Stock volatility and stroke mortality in a Chinese population. J Cardiovasc Med (Hagerstown). 2013; 14(9): 617-621. https://doi.org /10.2459/ JCM.0b013e32835ec51f .

2. Losey $P$, Ladds $E$, Laprais $M$, et al. The role of PPAR activation during the systemic response to brain injury. J Neuroinflammation. 2015;12(99): 1-10. https://doi.org /10. 1186/ s129 74-015-0295-7.

3. GBD 2016 Stroke Collaborators. Global, regional, and national burden of stroke, 1990-2016: a systematic analysis for the Global Burden of Disease Study 2016. Lancet Neurol. 2019;18(5):439458. https://doi.org/10.1016/ S1474-4422 (19) 300 34-1.

4. Feigin VL, Krishnamurthi RV, Parmar P. Update on the global burden of ischemic and hemorrhagic stroke in 1990-2013: the GBD 2013 study. Neuroepidemiology. 2015;45(3):161-176. https://doi.org/ $10.1159 / 000441085$.

5. Wang S, Shen B, Wei L, et al.ASsociation between socioeconomic status and prognosis after ischemic stroke in South China. Neurol Res. 2019;17:1-7. https://doi.org/10.1080/01616412.2019.1630165.

6. Gao Y, Jiang B, Sun H, et al. The burden of stroke in China: Results from a nationwide populationbased epidemiological survey. PLoS One.2018; 13(12): e0208 398. https://doi.org/10.1371/ journal.pone.020 8398.

7. Kluding PM, Tseng BY, Billinger SA. Exercise and executive function in individuals with chronic stroke: a pilot study. J Neurol Phys Ther. 2011;35(1):11. https: // doi.org / 10.1097/NPT.0b013e318208ee6c. 
8. Ning Gao, Tie Guo, Han Luo, et al. Association of the MMP-9 polymorphism and ischemic stroke risk in southern Chinese Han population. BMC Neurology, 2019,19(1):67. https: // doi.org / 10.1186/s12883-019-1285-7.

9. Neves AL, Emery N, Roussel R, et al. Allelic variations in superoxide dismutase-1 (SOD1) gene and renal and cardiovascular morbidity and mortality in type 2 diabetic subjects. Mol Genet Metab.2012; 106(3):359-365. https://doi.org/ 10.1016/ j.ymgme. 2012.04.023.

10. Hare JM. Oxidative stress and apoptosis in heart failure progression. Circ Res.2011; 89(3): 198-200.

11. Burgoyne JR, Mongue-Din H, Eaton P,et al. Redox signaling in cardiac physiology and pathology. Circ Res. 2012;111(8): 1091-1106. https://doi.org/ 10.1161/CIRCRESAHA.111.255216.

12. Zhou L, Xiang W, Potts $\mathrm{J}$, et al. Reduction in extracellular superoxide dismutase activity in AfricanAmerican patients with hypertension. Free Radic Biol Med. 2006;41(9): 1384-1391. https://doi.org/ 10.1016/j. freeradbiomed. 2006.07.019.

13. Faraci FM, Didion SP. Vascular protection: superoxide dismutase isoforms in the vessel wall, Arterioscler. Thromb Vasc Biol,2004;24(8):1367-1373. https://doi.org/ 10.1161/01.ATV.0000133604.20182.cf.

14. Miller AA, Budzyn K,Sobey CG. Vascular dysfunction in cerebrovascular disease: mechanisms and therapeutic intervention. Clin Sci (Lond). 2010;119(1): 1-17. https://doi.org/ 10.1042/CS20090649.

15. Tiwari MK, Hägglund PM, Møller IM,et al.Copper ion / H2O2 oxidation of Cu/ZnSuperoxidedismutase: Implications for enzymatic activity and antioxidant action. Redox Biol.2019;26:101262.https://doi.org/10.1016/j.redox.2019.101262.

16. Ray PD, Huang BW, Tsuji Y. Reactive oxygen species(ROS) homeostasis and redox regulation in cellular signaling. Cell Signal. 2012 ;24(5): 981-990. https://doi.org/ 10.1016/j.cellsig.2012.01.008.

17. Son $\mathrm{M}$, Cloyd CD, Rothstein JD,et al. Aggregate formation in $\mathrm{Cu}, \mathrm{Zn}$ superoxide dismutase-related proteins. J Biol Chem. 2003;278(16): 14331-14336. https://doi.org/ 10.1074/jbc.M211698200.

18. Alachkar H, Fulton N, Sanford B, et al. Expression and polymorphism (rs4880) of mitochondrial superoxide dismutase (SOD2) and asparaginase induced hepatotoxicity in adult patients with acute lymphoblastic leukemia. Pharmacogenomics. 2017;17(3):274-279. https://doi.org/ 10.1038/tpj.2016.7.

19. Ghattas MH, Abo-Elmatty DM.Association of polymorphic markers of the catalase and superoxide dismutase genes with type 2 diabetes mellitus.DNA Cell Biol. 2012;31 (11):1598-15603. https://doi.org/ 10.1089/dna.2012.1739.

20. Otaki Y, Watanabe T, Nishiyama S, et al. The Impact of Superoxide Dismutase-1 Genetic Variation on Cardiovascular and AllCause Mortality in a Prospective Cohort Study: The Yamagata (Takahata) Study. PLoS One. 2016;11(10): e0164732. https://doi.org/ 10.1371/journal.pone.0164732.

21. He YT, Tang BS, Cai ZL, et al.Effects of Fluoxetine on Neural Functional Prognosis after Ischemic Stroke : A Randomized Controlled Study in China. J Stroke Cerebrovasc Dis. 2016; 25(4):761-770. https://doi.org/ 10.1016/j.jstrokecerebrovasdis. 2015.11. 035. 
22. Kumar A, Misra S, Sagar R, et al. Relationship between Factor V Leiden Gene Variant and Risk of Ischemic Stroke: A Case-Control Study[J].Ann Indian Acad Neu 2017;20(3): 284-288. https://doi.org/ 10.4103/aian.AIAN_31_17.

23. Granger DN,Kvietys PR. Reperfusion injury and reactive oxygen species: The evolution of a concept. Redox Biol.2015;6: 524-551. https://doi.org/ 10.1016/j.redox.2015.08.020.

24. Broughton BR, Reutens DC, Sobey CG. Apoptotic mechanisms after cerebral ischemia. Stroke. 2009;40(5): 331-339. https://doi.org/ 10.1161/STROKEAHA. 108.531632.

25. Qiu HM, Yang JX,Jiang QS, et al.Inconsistency between manganese superoxide dismutase expression and its activity involved in the degeneration of recognition function induced by chronic aluminum overloading in mice. Hum Exp Toxicol. 2016;35(1):63-68. https://doi.org/ $10.1177 / 0960327115577522$.

26. Liu Y, Zha L, Li B, et al. Correlation between superoxide dismutase 1 and 2 polymorphisms and susceptibility to oral squamous cell carcinoma. Exp Ther Med. 2014;7(1):171-178. https://doi.org/ 10.3892/etm. 2013.1375.

27. Fazakas A, Szelenyi Z,Szenasi G, et al. Genetic predisposition in patients with hypertension and normal ejection fraction to oxidative stress.J Am Soc Hypertens.2016; 10(2): 124-132. https:// doi. org/ 10.1016/ j.jash. 2015.11.013.

28. Wiener HW,Perry RT,Chen Z, et al.A polymorphism in SOD2 is associated with development of Alzheimer's disease. Genes Brain Behav.2007;6(8):770-775. https://doi.org/ 10.1111/j.1601- 183X. 2007.00308.x.

29. Tamai $\mathrm{M}$, Furuta $\mathrm{H}$,Kawashima $\mathrm{H}$, et al.Extracellular superoxide dismutase gene polymorphism is associated with insulin resistance and the susceptibility to type 2 diabetes.Diabetes Res Clin Pract,2006,71(2):140-145. https://doi.org/ 10.1016/j.diabres. 20 05.05.006.

30. Takahiro N,Tomohiro N, Sato N, et al.Association of extracellular superoxide dismutase gene with cerebral infarction in women: a haplotype-based case-control study. Hereditas, 2008;145( 6) : 283 -292. https://doi.org/ 10.1111/j.1601-5223. 2008.02 086.x 